\title{
Chronic Peritoneal Effusion
}

National Cancer Institute

\section{Source}

National Cancer Institute. Chronic Peritoneal Effusion. NCI Thesaurus. Code C34920.

Persistent or recurring accumulation of fluid in the peritoneal cavity. 